\title{
An algorithm for computing the distance to uncontrollability
}

\author{
L. Elsner and C. $\mathrm{He}^{*}$ \\ Fakultät fur Mathematik, Universität Bielefeld, Postfach 8640, 4800 Bielefeld 1, Germany
}

Received 25 February 1991

Revised 18 May 1991

Abstract: In this paper, we present an algorithm to compute the distance to uncontrollability. The problem of computing the distance is an optimization problem of minimizing $\sigma(x, y)$ over the complete plane. This new approach is based on finding zero points of grad $\sigma(x, y)$. We obtain the explicit expression of the derivative matrix of $\operatorname{grad} \sigma(x, y)$. The Newton's method and the bisection method are applied to approach these zero points. Numerical results show that these methods work well.

Keywords: Controllability; distance to uncontrollability; singular value decomposition; Newton's method.

\section{Introduction}

One of the fundamental concepts in linear control theory is that of controllability. A pair $(A, B)$ of matrices $A \in R^{n \times n}, B \in R^{n \times m}$ is controllable if in the system

$$
\dot{x}=A x+B u,
$$

for any initial state $x_{0}$, final state $x_{1}$ and any $t_{1}>0$, there is a continuous function $u(t)$ such that the solution of (1.1) with $x(0)=x_{0}$ satisfies $x\left(t_{1}\right)=x_{1}$. It is well known that $(A, B)$ is controllable iff

$$
\operatorname{rank}([A-s I, B])=n, \forall s \in C \text {. }
$$

In [9], Paige defined the 'distance to uncontrollability' as the spectral norm distance of the pair $(A, B)$ from the set of all uncontrollable pairs:

$$
d(A, B)=\min \{\|[E, F]\|:(A+E, B+F) \text { uncontrollable }\},
$$

where \|\| denotes the spectral norm, and $[E, F]$ is the $n \times(n+m)$ matrix formed by the columns of $E$ followed by those of $F$. It was pointed out by Eising [5,6] that $d(A, B)$ admits the following description

$$
d(A, B)=\min _{s \in C} \sigma_{n}([A-s I, B])=\min _{s \in C} \sigma(s),
$$

where $\sigma_{n}(G)$ denotes the $n$-th singular value of a $n \times(n+m)$ matrix $G$. It is clear that the problem of finding the distance to uncontrollability is the problem of minimizing $\sigma(s)$ over the complex plane.

Another characterization of $d(A, B)$ is given by

$$
d(A, B)=\min \left\{\left\|q^{\mathrm{H}}\left[A-q^{\mathrm{H}} A q I, B\right]\right\|:\|q\|=1\right\},
$$

where \|\| is the Euclidean vector norm [5,12]. There are several algorithms in the literature for calculating $d(A, B)$. They are based on the minimization of $\sigma(s)$. Their main drawback is that they need a good starting point to converge $[2,4,5,12]$. Here we propose to use Newton's method with damping.

* Supported by the Alexander von Humboldt research foundation. 
This method is known to show convergence also for not so good starting values, a behaviour observed in our examples too.

We are able to use this method, because we can explicitly calculate the first and the second partial derivatives of $\sigma(x, y)=\sigma(x+\mathrm{i} y)=\sigma(s)$ using the singular value decomposition (SVD) of $[A-s I, B]$. Let

$$
[A-s I, B]=U \Sigma V^{\mathrm{H}}
$$

be the SVD, where $\Sigma$ is the $n \times(m+n)$ diagonal matrix with diagonal elements $\sigma_{1} \geqslant \sigma_{2} \geqslant \cdots \geqslant \sigma_{n} \geqslant 0$, and $U$ and $V$ are the $n \times n$ resp. $(m+n) \times(m+n)$ unitary matrices, the columns of which are the left resp. right normalized singular vectors of $[A-s I, B]$. If $\sigma_{n}$ is a simple singular value then the normalized left singular vector $u_{n}(s)$ (the $n$-th column of $U$ ), and the normalized right vector $v_{n}(s)$ (the $n$-th column of $V$ ) are uniquely determined by (1.6) up to a common factor and so

$$
f(s)=v_{n}^{\mathrm{H}}(s)\left(\begin{array}{c}
u_{n}(s) \\
0
\end{array}\right)
$$

is well defined. This function plays an important role, it is shown that

$$
\frac{\partial \sigma(x+\mathrm{i} y)}{\partial x}=-\operatorname{Re} f(x+\mathrm{i} y), \frac{\partial \sigma(x+\mathrm{i} y)}{\partial y}=-\operatorname{Im} f(x+\mathrm{i} y),
$$

and hence the zero points of $f(s)$ are the critical points of the function $\sigma(s)$. In addition, we have

$$
\sigma(s) f(s)=u_{n}^{\mathrm{H}}(s)(A-s I) u_{n}(s),
$$

as $[A-s I, B]{ }^{\mathrm{H}} u_{n}(s)=\sigma(s) v_{n}(s)$. This shows that the critical points satisfy $s=u_{n}^{\mathrm{H}}(s) A u_{n}(s)$, and hence lie in the field of values of $A$.

The paper is organised as follows. In Section 2 we study the function $\sigma(x, y)=\sigma(x+i y)$. It is analytic as a function of the real parameters $x$ and $y$ for all but a finite number of points. We calculate the first and second derivatives of $\sigma(x, y)$ using an SVD. Here we treat a slightly more general case. In Section 3, the connection between zeros of $f(s)$ and local minima of $\sigma(x, y)$ is studied. Two criteria, one analytic and one in matrix terms, are given which guarantee a critical point of $\sigma(x, y)$ to be a local minimum. In Section 4 several possibilities of using Newton's method are outlined. They are the cases of real or complex parameters $s$. Numerical results and some discussions concerning the case of multiple singular values are given in Section 5 and 6 respectively.

\section{The explicit expressions of the first and second derivatives of $\sigma(x, y)$}

In this section, the main results are the explicit expressions of the first and second derivatives of $\sigma(x, y)$ given in (2.10) and (2.11). Let us consider a more general case of a complex matrix $G(s)=G_{1}+$ $s G_{2}$ with a real parameter $s$. It is well known that a singular value of $G(s)$ is analytic if it is simple [13]. In the following theorem, we give explicit expressions of its first and second derivatives. More general results can be found in $[10,11]$.

Theorem 1. Let $G(s)=G_{1}+s G_{2}$ be an $n \times p$ complex matrix $(n \leq p)$ with a real parameter $s$, and $G(s)=U(s) \Sigma(s) V(s)^{\mathrm{H}}$ be the SVD of $G(s)$ with the last singular value $\sigma_{n}(s)=\sigma(s)$ being simple, then

$$
\dot{\sigma}=\frac{\mathrm{d} \sigma}{\mathrm{d} s}=\operatorname{Re}\left(v_{n}^{\mathrm{H}}(s) G_{2}^{\mathrm{H}} u_{n}(s)\right)
$$


and

$$
\begin{aligned}
\ddot{\sigma}=\frac{\mathrm{d}^{2} \sigma}{(\mathrm{d} s)^{2}}= & \operatorname{Re}\left(v_{\mathrm{d} s}^{\mathrm{H}} G_{2}^{\mathrm{H}} u_{n}(s)+v_{n}^{\mathrm{H}}(s) G_{2}^{\mathrm{H}} u_{\mathrm{d} s}\right) \\
& +\operatorname{Re}\left(-\frac{\mathrm{i}}{\sigma_{n}(s)} \operatorname{Im}\left(v_{n}^{\mathrm{H}}(s) G_{2}^{\mathrm{H}} u_{n}(s)\right) v_{n}^{\mathrm{H}}(s) G_{2}^{\mathrm{H}} u_{n}(s)\right),
\end{aligned}
$$

where $u_{\mathrm{d} s}$ and $v_{\mathrm{d} s}$ are given in (2.3) and (2.4).

Before proving Theorem 1, we will prove Lemma 2, which gives formulas for $\dot{u}_{n}(s)$ and $\dot{v}_{n}(s)$ in terms of the $u_{j}(s), v_{j}(s)$ and $h_{u}(s)=u_{n}^{\mathrm{H}}(s) \dot{u}_{n}(s), h_{v}(s)=v_{n}^{\mathrm{H}}(s) \dot{v}_{n}(s)$.

Lemma 2. Under the assumptions of Theorem 1, the derivatives of $u_{n}(s)$ and $v_{n}(s)$ satisfy

$$
\begin{aligned}
& \dot{u}_{n}(s)=u_{\mathrm{d} s}+h_{u}(s) u_{n}(s), \\
& \dot{v}_{n}(s)=v_{\mathrm{d} s}+h_{r}(s) v_{n}(s),
\end{aligned}
$$

where $u_{\mathrm{d} s}=-\sum_{j=1}^{n-1} \alpha_{j}(s) u_{j}(s)$ and

$$
v_{\mathrm{d} s}=-\sum_{j=1}^{n-1} \beta_{j}(s) v_{j}(s)+\frac{1}{\sigma_{n}(s)} \sum_{j=n+1}^{p}\left(v_{j}^{\mathrm{H}}(s) \dot{G}^{\mathrm{H}}(s) u_{n}(s)\right) v_{j}(s)
$$

with

$$
\begin{aligned}
& \alpha_{j}(s)=\frac{\sigma_{n}(s) u_{j}^{\mathrm{H}}(s) \dot{G}(s) v_{n}(s)+\sigma_{j}(s) v_{j}^{\mathrm{H}}(s) \dot{G}^{\mathrm{H}}(s) u_{n}(s)}{\sigma_{j}^{2}(s)-\sigma_{n}^{2}(s)}, \\
& \beta_{j}(s)=\frac{\sigma_{j}(s) u_{j}^{\mathrm{H}}(s) \dot{G}(s) v_{n}(s)+\sigma_{n}(s) v_{j}^{\mathrm{H}}(s) \dot{G}^{\mathrm{H}}(s) u_{n}(s)}{\sigma_{j}^{2}(s)-\sigma_{n}^{2}(s)},
\end{aligned}
$$

and $h_{u}(s)=u_{n}^{\mathrm{H}}(s) \dot{u}_{n}(s), h_{\imath}(s)=v_{n}^{\mathrm{H}}(s) \dot{v}_{n}(s)$. The last two functions satisfy

$\operatorname{Re} h_{u}(s)=0, \quad \operatorname{Re} h_{c}(s)=0$,

$$
h_{u}(s)+\bar{h}_{i}(s)=-\frac{\mathrm{i}}{\sigma_{n}(s)} \operatorname{Im}\left(v_{n}^{\mathrm{H}}(s) \dot{G}^{\mathrm{H}}(s) u_{n}(s)\right) \text {. }
$$

Remark. Observe that $h_{u}$ and $h_{v}$ contain the derivatives too.

Proof and Lemma 2. In the following proof, we omit the parameter $s$. So keep in mind that all the mentioned vectors and matrices are functions of $s$. According to the SVD of $G$, we have

$$
G G^{\mathrm{H}} u_{n}=\sigma_{n}^{2} u_{n} .
$$

It is well-known that the eigenvalues and the eigenvectors of $G G^{\mathrm{H}}$ are analytic with respect to the real parameter $s$ if the eigenvalues are simple [13]. So the derivative of $u_{n}$ satisfies

$$
\left(G G^{\mathrm{H}}-\sigma_{n}^{2} I\right) \dot{u}_{n}=-\dot{G} G^{\mathrm{H}} u_{n}-G \dot{G}^{\mathrm{H}} u_{n}+2 \sigma_{n} \dot{\sigma}_{n} u_{n} .
$$

Thus from $G=U \Sigma V^{\mathrm{H}}$,

$$
\left(\Sigma \Sigma^{\mathrm{H}}-\sigma_{n}^{2} I\right) U^{\mathrm{H}} \dot{u}_{n}=-\sigma_{n} U^{\mathrm{H}} \dot{G} v_{n}-\Sigma V^{\mathrm{H}} \dot{G}^{\mathrm{H}} u_{n}+2 \sigma_{n} \dot{\sigma}_{n} e_{n},
$$


i.e. the first $n-1$ equations in

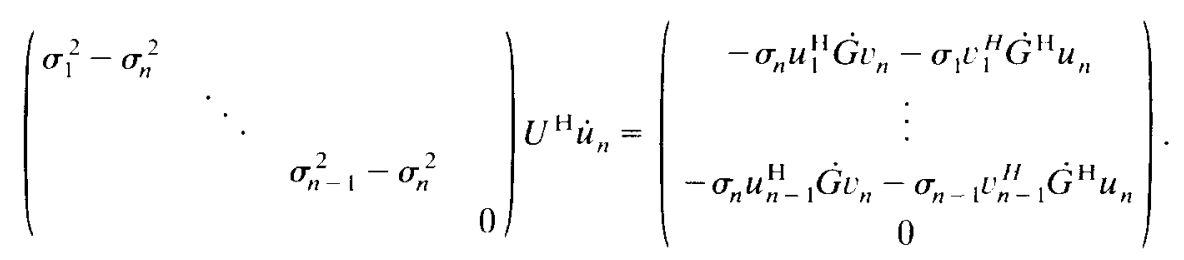

The last equation is just $u_{n}^{\mathrm{H}} \dot{u}_{n}=h_{u}$. Solving for $U^{\mathrm{H}} \dot{u}_{n}$ gives (2.3) and similarly (2.4) is obtained by applying the same kind of analysis of $G^{\mathrm{H}} G v_{n}=\sigma_{n}^{2} v_{n}$. Now we consider the properties of $h_{u}$ and $h_{\imath}$. Note that $u_{n}^{\mathrm{H}} u_{n}=1$, so $u_{n}^{\mathrm{H}} \dot{u}_{n}+\dot{u}_{n}^{\mathrm{H}} u_{n}=0$, i.e. $\operatorname{Re} h_{u}=0$ and $\operatorname{Re} h_{r}=0$. Formula (2.5) follows from the observation that $\sigma_{n}=v_{n}^{\mathrm{H}} G^{\mathrm{H}} u_{n}$ and

$$
\dot{\sigma}_{n}=v_{n}^{\mathrm{H}} \dot{G}^{\mathrm{H}} u_{n}+\sigma_{n}\left(h_{u}+\bar{h}_{c}\right)
$$

As $\dot{\sigma}_{n}$ is real, and $h_{u}+\bar{h}_{l}$ is purely imaginary, we have

$$
h_{u}+\bar{h}_{r}=-\frac{\mathrm{i}}{\sigma_{n}} \operatorname{Im}\left(v_{n}^{\mathrm{H}} \dot{G}^{\mathrm{H}} u_{n}\right) \text {. }
$$

Proof of Theorem 1. From Lemma 2, it is easy to prove the conclusions of Theorem 1. As a direct consequence of (2.6), we obtain (2.1) as $\dot{G}=G_{2}$. Now we differentiate (2.1). Replacing $\dot{u}_{n}$ and $\dot{i}_{n}$ by (2.3) (2.4), we see that the unknown terms $h_{u}$ and $h_{r}$ appear only in the form $h_{u}+\bar{h}_{r}$ and can be replaced by (2.7). Thus we get (2.2).

Let us come back to our original problem. We consider first the real case $G(x)=[A-x I, B]$ and $\sigma_{n}(x), u_{n}(x)$ and $v_{n}(x)$ are all real. It is easy to see from Lemma 2 that both $h_{u}(x)$ and $h_{i}(x)$ vanish. In view of $\dot{G}(x)=-[I, 0]$, Theorem 1 gives now Corollary 3 .

Corollary 3. Let $x$ be a real parameter and $[A-x I, B]=U(x) \Sigma(x) V^{\mathrm{T}}(x)$ be the SVD of $[A-x I, B]$ with $\sigma_{n}(x)=\sigma(x)$ being simple and $f(x)=v_{n}^{\mathrm{T}}(x)\left({ }_{0}^{u_{n}(x)}\right)$, then the first and second derivatives of $\sigma(x)$ are given by

$$
\frac{\mathrm{d} \sigma}{\mathrm{d} x}=-f(x)
$$

and

$$
\frac{\mathrm{d}^{2} \sigma}{\mathrm{d} x^{2}}=-\dot{f}(x)=-\left(\dot{v}_{n}^{\mathrm{T}}(x)\left(\begin{array}{c}
u_{n}(x) \\
0
\end{array}\right)+v_{n}^{\mathrm{T}}(x)\left(\begin{array}{c}
\dot{u}_{n}^{\mathrm{T}}(x) \\
0
\end{array}\right)\right)
$$

Here

$$
\dot{u}_{n}(x)=\sum_{j=1}^{n-1} \alpha_{j}(x) u_{j}(x), \quad \dot{v}_{n}(x)=\sum_{j=1}^{n-1} \beta_{j}(x) v_{j}(x)-\frac{1}{\sigma_{n}(x)} \sum_{j=n+1}^{n+m}\left(v_{j}^{\mathrm{T}}(x)\left(\begin{array}{c}
u_{n}(s) \\
0
\end{array}\right)\right) v_{j}(x),
$$

where

$$
\begin{gathered}
\alpha_{j}(x)=\frac{\sigma_{n}(x)\left[u_{j}^{\mathrm{T}}(x), 0\right] v_{n}(x)+\sigma_{j}(x) v_{j}^{\mathrm{T}}(x)\left(\begin{array}{c}
u_{n}(x) \\
0
\end{array}\right)}{\sigma_{j}^{2}(x)-\sigma_{n}^{2}(x)}, \\
\beta_{j}(x)=\frac{\sigma_{j}(x)\left[u_{j}^{\mathrm{T}}(x), 0\right] v_{n}(x)+\sigma_{n}(x) v_{j}^{\mathrm{T}}(x)\left(\begin{array}{c}
u_{n}(x) \\
0
\end{array}\right)}{\sigma_{j}^{2}(x)-\sigma_{n}^{2}(x)} .
\end{gathered}
$$


Now we consider the case of the complex parameter $s=x+\mathrm{i} y$. Note that $G(x, y)=[A-(x+$ i $y) I, B], \partial G(x, y) / \partial x=-[I, 0]$ and $\partial G(x, y) / \partial y=-\mathrm{i}[I, 0]$. Substituting these two partial derivatives to $G_{2}$ in (2.1) and (2.2), Theorem 1 gives the formulas of the partial derivatives of $\sigma(x, y)$.

Corollary 4. Let $[A-(x+\mathrm{i} y) I, B]=U(x, y) \Sigma(x, y) V(x, y)^{\mathrm{H}}$ be the SVD of $[A-(x+\mathrm{i} y) I, B]$ with $\sigma_{n}(x, y)=\sigma(x, y)$ being simple and $f(x, y)=f(s)$ is defined in $(1.7)$, then we have

$$
\begin{aligned}
& \frac{\partial \sigma}{\partial x}=-\operatorname{Re} f, \quad \frac{\partial \sigma}{\partial y}=-\operatorname{Im} f \\
& \frac{\partial^{2} \sigma}{\partial x^{2}}=-\operatorname{Re} \frac{\partial f}{\partial x}, \quad \frac{\partial^{2} \sigma}{\partial x \partial y}=-\operatorname{Re} \frac{\partial f}{\partial y}, \quad \frac{\partial^{2} \sigma}{\partial y^{2}}=-\operatorname{Im} \frac{\partial f}{\partial y} .
\end{aligned}
$$

Here

$$
\frac{\partial f}{\partial x}=v_{\mathrm{d} x}^{\mathrm{H}}\left(\begin{array}{c}
u_{n} \\
0
\end{array}\right)+v_{n}^{\mathrm{H}}\left(\begin{array}{c}
u_{\mathrm{d} x} \\
0
\end{array}\right)+\frac{\mathrm{i}}{\sigma_{n}}(\operatorname{Im} f) f,
$$

where $u_{\mathrm{d} x}$ and $v_{\mathrm{d}} x$ are given in (2.14) and (2.15).

$$
\frac{\partial f}{\partial y}=v_{\mathrm{d} y}^{\mathrm{H}}\left(\begin{array}{c}
u_{n} \\
0
\end{array}\right)+v_{n}^{\mathrm{H}}\left(\begin{array}{c}
u_{\mathrm{d} y} \\
0
\end{array}\right)-\frac{\mathrm{i}}{\sigma_{n}}(\operatorname{Re} f) f,
$$

where $u_{\mathrm{d} y}$ and $v_{\mathrm{d} y}$ are given in (2.16) and (2.17).

$$
\begin{aligned}
& u_{\mathrm{d} x}=\sum_{j=1}^{n-1} \alpha_{x j} u_{j}, \\
& v_{\mathrm{d} x}=\sum_{j=1}^{n-1} \beta_{x j} v_{j}-\frac{1}{\sigma_{n}} \sum_{j=n+1}^{n+m}\left(v_{j}^{\mathrm{H}}\left(\begin{array}{c}
u_{n} \\
0
\end{array}\right)\right) v_{j},
\end{aligned}
$$

where

$$
\begin{aligned}
& \alpha_{x j}=\frac{\sigma_{n}\left[u_{j}^{\mathrm{H}}, 0\right] v_{n}+\sigma_{j} v_{j}^{\mathrm{H}}\left(\begin{array}{c}
u_{n} \\
0
\end{array}\right)}{\sigma_{j}^{2}-\sigma_{n}^{2}}, \quad \beta_{x j}=\frac{\sigma_{j}\left[u_{j}^{\mathrm{H}}, 0\right] v_{n}+\sigma_{n} v_{j}^{\mathrm{H}}\left(\begin{array}{c}
u_{n} \\
0
\end{array}\right)}{\sigma_{j}^{2}-\sigma_{n}^{2}} . \\
& u_{\mathrm{d} y}=\mathrm{i} \sum_{j=1}^{n-1} \alpha_{y j} u_{j}, \\
& v_{\mathrm{d} y}=\mathrm{i} \sum_{j=1}^{n-1} \beta_{y j} v_{j}+\frac{\mathrm{i}}{\sigma_{n}} \sum_{j=n+1}^{n+m}\left(v_{j}^{\mathrm{H}}\left(\begin{array}{c}
u_{n} \\
0
\end{array}\right)\right) v_{j},
\end{aligned}
$$

where

$$
\alpha_{y j}=\frac{\sigma_{n}\left[u_{j}^{\mathrm{H}}, 0\right] v_{n}-\sigma_{j} v_{j}^{\mathrm{H}}\left(\begin{array}{c}
u_{n} \\
0
\end{array}\right)}{\sigma_{j}^{2}-\sigma_{n}^{2}}, \quad \beta_{y j}=\frac{\sigma_{j}\left[u_{j}^{\mathrm{H}}, 0\right] v_{n}-\sigma_{n} v_{j}^{\mathrm{H}}\left(\begin{array}{c}
u_{n} \\
0
\end{array}\right)}{\sigma_{j}^{2}-\sigma_{n}^{2}} .
$$

\section{The local minimum of $\sigma(x, y)$}

From the nice relation between $\operatorname{grad} \sigma(x, y)$ and $f(x, y)(2.10)$, we conclude the following result. 
Theorem 5. $s^{*}=x^{*}+\mathrm{i} y^{*}$ is a zero point of $f(s)$ defined in (1.7) iff $\left(x^{*}, y^{*}\right)$ is a critical point of $\sigma(x, y)=\sigma_{n}[A-(x+\mathrm{i} y) I, B]$.

From this theorem, the computation of $d(A, B)$ is equivalent to find the zero points of $f(x, y)$, in which $-(\operatorname{Re} f(x, y), \operatorname{Im} f(x, y))^{\mathrm{T}}$ will be the gradient of $\sigma(x, y)$. The critical points of $\sigma(x, y)$ are divided into three groups, local minima, local maxima and saddle points.

Let $f\left(s^{*}\right)=0, s^{*}=x^{*}+\mathrm{i} y^{*}$. The following is well known.

(a) If $\left(\partial^{2} \sigma / \partial x^{2}\right)\left(\partial^{2} \sigma / \partial y^{2}\right)-\left(\partial^{2} \sigma / \partial x \partial y\right)^{2}>0$ and $\partial^{2} \sigma / \partial x^{2}<0$, then $\left(x^{*}, y^{*}\right)$ is a local maximum of $\sigma(x, y)$.

(b) If $\left(\partial^{2} \sigma / \partial x^{2}\right)\left(\partial^{2} \sigma / \partial y^{2}\right)-\left(\partial^{2} \sigma / \partial x \partial y\right)^{2}>0$ and $\partial^{2} \sigma / \partial x^{2}>0$, then $\left(x^{*}, y^{*}\right)$ is a local minimum of $\sigma(x, y)$.

(c) If $\left(\partial^{2} \sigma / \partial x^{2}\right)\left(\partial^{2} \sigma / \partial y^{2}\right)-\left(\partial^{2} \sigma / \partial x \partial y\right)^{2}<0$, then $\left(x^{*}, y^{*}\right)$ is a saddle point of $\sigma(x, y)$.

Using the results of Corollary 4 , we can now decide to which group the critical points $s^{*}$ belongs. We can also give a sufficient condition of $\left(x^{*}, y^{*}\right)$ being a local minimum of $\sigma(x, y)$ in matrix theoretic terms.

Theorem 6. If $s^{*}=x^{*}+\mathrm{i} y^{*}$ is a zero point of $f(s), u_{n}^{*}=u_{n}\left(x^{*}, y^{*}\right)$ and

$$
\sigma_{n-1}^{2}\left[A-s^{*} I, B\right]-\sigma_{n}^{2}\left[A-s^{*} I, B\right]>4\left\|u_{n}^{* \mathrm{H}}\left(A-s^{*} I\right)\right\|^{2},
$$

then $\left(x^{*}, y^{*}\right)$ is a local minimum of $\sigma(x, y)$.

Proof. Let $s=x+\mathrm{i} y=s^{*}+\delta$ be a point near to $s^{*}$ and $q=u_{n}(x, y)$ be the $n$-th left singular vector of $[A-s I, B]$ corresponding to $\sigma_{n}(x, y)$. Then we have

$$
\begin{aligned}
\| q^{\mathrm{H}} & {\left[A-q^{\mathrm{H}} A q I, B\right] \|^{2} } \\
= & q^{\mathrm{H}}\left[A-s^{*} I, B\right]\left[A-s^{*} I, B\right]^{\mathrm{H}} q \\
& +q^{\mathrm{H}}\left(\left[A-q^{\mathrm{H}} A q I, B\right]\left[A-q^{\mathrm{H}} A q I, B\right]-\left[A-s^{*} I, B\right]\left[A-s^{*} I, B\right]^{\mathrm{H}}\right) q \\
= & q^{\mathrm{H}}\left[A-s^{*} I, B\right]\left[A-s^{*} I, B\right]^{\mathrm{H}} q-\left|q^{\mathrm{H}}\left(A-s^{*} I\right) q\right|^{2} .
\end{aligned}
$$

Write $q=a u_{n}^{*}+b h$ with $h^{\mathrm{H}} u_{n}^{*}=0,\|h\|=1,|a|^{2}+|b|^{2}=1$. Thus according to perturbation theory for eigenvectors using the simplicity of $\sigma_{n}$, one has $|b|=\mathrm{O}(\delta)$. Note that

$$
\begin{aligned}
q^{\mathrm{H}}\left[A-s^{*} I, B\right]\left[A-s^{*} I, B\right]^{\mathrm{H}} q & =|a|^{2} \sigma_{n}^{2}+|b|^{2} h^{\mathrm{H}}\left[A-s^{*} I, B\right]\left[A-s^{*} I, B\right]^{\mathrm{H}} h \\
& =\sigma_{n}^{2}+|b|^{2}\left(h^{\mathrm{H}}\left[A-s^{*} I, B\right]\left[A-s^{*} I, B\right] h-\sigma_{n}^{2}\right)
\end{aligned}
$$

and

$$
\begin{aligned}
\left|q^{\mathrm{H}}\left(A-s^{*} I\right) q\right|^{2} & =\left|\bar{b} a h^{\mathrm{H}}\left(A-s^{*} I\right) u_{n}^{*}+\bar{a} b u_{n}^{* \mathrm{H}}\left(A-s^{*} I\right) h\right|^{2}+\mathrm{O}\left(\delta^{3}\right) \\
& =\left|2 \operatorname{Re} \bar{a} b u_{n}^{* \mathrm{H}}\left(A-s^{*} I\right) h\right|^{2}+\mathrm{O}\left(\delta^{3}\right) .
\end{aligned}
$$

Thus

$$
\begin{aligned}
\| & q^{\mathrm{H}}\left[A-q^{\mathrm{H}} A q I, B\right] \|^{2}-\sigma_{n}^{2}\left(x^{*}, y^{*}\right) \\
& =|b|^{2}\left(h^{\mathrm{H}}\left[A-s^{*} I, B\right]\left[A-s^{*} I, B\right]^{\mathrm{H}} h-\sigma_{n}^{2}\right)-\left|2 \operatorname{Re} \bar{a} b u_{n}^{* H}\left(A-s^{*} I\right) h\right|^{2}+\mathrm{O}\left(\delta^{3}\right) \\
& \geq|b|^{2}\left(\sigma_{n-1}^{2}-\sigma_{n}^{2}\right)-4|a|^{2}|b|^{2}\left\|u_{n}^{* \mathrm{H}}\left(A-s^{*} I\right)\right\|^{2}+\mathrm{O}\left(\delta^{3}\right) \\
& =|b|^{2}\left(\sigma_{n-1}^{2}-\sigma_{n}^{2}-4\left\|u_{n}^{* H}\left(A-s^{*} I\right)\right\|^{2}\right)+\mathrm{O}\left(\delta^{3}\right) .
\end{aligned}
$$


According to the condition of (3.1), $\left\|q^{\mathrm{H}}\left[A-q^{\mathrm{H}} A q I, B\right]\right\|^{2} \geq \sigma_{n}^{2}\left(x^{*}, y^{*}\right)$ is always true. Since

$$
\sigma_{n}[A-(x+\mathrm{i} y) I, B]=\left\|q^{\mathrm{H}}[A-(x+\mathrm{i} y) I, B]\right\| \geq\left\|q^{\mathrm{H}}\left[A-q^{\mathrm{H}} A q I, B\right]\right\|,
$$

$\sigma_{n}(x, y) \geq \sigma_{n}\left(x^{*}, y^{*}\right)$. Thus $\left(x^{*}, y^{*}\right)$ is a local minimum of $\sigma_{n}(x, y)$.

Since

$$
\left\|u_{n}^{\mathrm{H}}\left(A-s^{*} I\right)\right\|=\left\|u_{n}^{\mathrm{H}}\left[A-s^{*} I, B\right]\left(\begin{array}{l}
I \\
0
\end{array}\right)\right\| \leq \sigma_{n}\left(x^{*}, y^{*}\right),
$$

we get at once the following sufficient condition.

Corollary 7. If $s^{*}=x^{*}+\mathrm{i} y^{*}$ satisfies $f\left(s^{*}\right)=0$ and

$$
\sigma_{n-1}\left[A-s^{*} I, B\right]>\sqrt{5} \sigma_{n}\left[A-s^{*} I, B\right],
$$

then $\left(x^{*}, y^{*}\right)$ is a local minimum point of $\sigma(x, y)$.

\section{Newton's algorithm}

Because we have obtained the first and second partial derivatives of $\sigma(x, y)$ in terms of the SVD of [ $A-s I, B]$ when $\sigma(x, y)$ is simple, Newton's method can be applied to compute the minimum points of $\sigma(x, y)$. Generally speaking, the local minima of $\sigma(x, y)$ happen when they are simple. More details are discussed in Section 6. As $u_{n}^{* \mathrm{H}} A u_{n}^{*}=s^{*}$, all minimum points $s^{*}=x^{*}+\mathrm{i} y^{*}$ lie in the field of values of $A$, and hence

$$
\lambda_{\min }\left(\frac{A+A^{\mathrm{T}}}{2}\right) \leq x^{*} \leq \lambda_{\max }\left(\frac{A+A^{\mathrm{T}}}{2}\right), \quad \lambda_{\min }\left(\frac{A-A^{\mathrm{T}}}{2 \mathrm{i}}\right) \leq y^{*} \leq \lambda_{\max }\left(\frac{A-A^{\mathrm{T}}}{2 \mathrm{i}}\right) .
$$

Here $\lambda_{\min }(A)$ and $\lambda_{\max }(A)$ denote the minimal and the maximal eigenvalue of $A$. Since $\sigma_{n}\left[A-s^{*} I, B\right]$ $=\sigma_{n}\left[A-\bar{s}^{*} I, B\right]$, the search for minimum points can be restricted to

$$
0 \leq y^{*} \leq \lambda_{\max }\left(\frac{A-A^{\mathrm{T}}}{2 \mathrm{i}}\right)
$$

Theorem 5 also suggests a method to compute $d(A, B)$. We need only to find all zeros of $f(s)$, which are the critical points of $\sigma(s)$. Especially in the case of $f(s)$ being a real function of a real parameter $s$ in order to compute the following $d_{\mathrm{r}}(A, B)$, the bisection method can be used to find all zeros of $f(x)$. We ought to say that Theorem 6 and Corollary 7 and the criterions of the second partial derivatives give only sufficient conditions to determine which zeros of $f(s)$ are local minima of $\sigma(s)$.

\subsection{Real case}

We first consider the problem of computing

$$
d_{\mathrm{r}}(A, B)=\min _{s \in R} \sigma_{n}[A-s I, B] .
$$

So $f(s)$ will be real. Since $s^{*}=u_{n}^{\mathrm{T}}\left(s^{*}\right) A u_{n}\left(s^{*}\right), s^{*}$ is in the interval

$$
I_{\mathrm{r}}=\left[\lambda_{\min }\left(\frac{A+A^{\mathrm{T}}}{2}\right), \lambda_{\max }\left(\frac{A+A^{\mathrm{T}}}{2}\right)\right] \text {. }
$$


Also since $u_{n}^{\top}(s) A u_{n}(s)-s=\sigma_{n}(s) f(s)$, we have

$$
f(s)>0 \text { for } s<\lambda_{\min }\left(\frac{A+A^{\mathrm{T}}}{2}\right), \quad f(s)<0 \text { for } s>\lambda_{\max }\left(\frac{A+A^{\mathrm{T}}}{2}\right) .
$$

The following Newton method is suggested to compute the minimum points of $\sigma(s)=\sigma_{n}(s)$.

Newton's algorithm (real case). Choose $s_{0} \in I_{r}$. For $k=1,2, \ldots$,

$$
s_{k+1}=s_{k}-\theta_{k} \frac{f\left(s_{k}\right)}{\dot{f}\left(s_{k}\right)}
$$

where $\theta_{k}$ is such that $\sigma\left(s_{k+1}\right)<\sigma\left(s_{k}\right)$.

In our examples a choice $\theta_{k} \neq 1$ is only neccessary at the beginning steps of the Newton algorithm. After having a good approximation of a local minimum point, we can take $\theta_{k}=1$ and hence have the usual Newton algorithm. Also the following bisection method can be used to find the zeros of $f(s)$.

Bisection method. (a) Find an interval $[a, b]$ such that $f(a) * f(b)<0$.

(b) Let $c=\frac{1}{2}(a+b)$, if $f(c) * f(b)<0$ then $a=c$ and go back $(b)$ and if $f(a) * f(c)<0$ then $b=c$ and go back $(b)$. The step (b) is repeated until $c$ is an acceptable zero point of $f(s)$.

\subsection{Complex case}

In order to compute $d(A, B)=\min _{s \in C} \sigma_{n}([A-s I, B])$, we have the following Newton algorithm for complex $s$.

Newton's algorithm (complex case). Choose $\left(\begin{array}{l}x_{01} \\ y_{0}\end{array}\right)$. For $k=1,2, \ldots$,

$$
\left(\begin{array}{l}
x_{k+1} \\
y_{k+1}
\end{array}\right)=\left(\begin{array}{l}
x_{k} \\
y_{k}
\end{array}\right)-\theta_{k}\left(\begin{array}{l}
p_{k 1} \\
p_{k 2}
\end{array}\right)
$$

where

$$
\left(\begin{array}{l}
p_{k 1} \\
p_{k 2}
\end{array}\right)=\left(\begin{array}{cc}
\operatorname{Re} \frac{\partial f}{\partial x} & \operatorname{Re} \frac{\partial f}{\partial y} \\
\operatorname{Im} \frac{\partial f}{\partial x} & \operatorname{Im} \frac{\partial f}{\partial y}
\end{array}\right)^{-1}\left(\begin{array}{l}
\operatorname{Re} f(x, y) \\
\operatorname{Im} f(x, y)
\end{array}\right),
$$

and $\theta_{k}$ such that

$$
\sigma\left(x_{k}-\theta_{k} p_{k 1}, y_{k}-\theta_{k} p_{k 2}\right)=\min _{-1 \leq \theta \leq 1} \sigma\left(x_{k}-\theta p_{k 1}, y_{k}-\theta p_{k 2}\right) \text {. }
$$

Computing the minimum of $\sigma\left(x_{k}-\theta p_{k 1}, y_{k}-\theta p_{k 2}\right)$ in $[-1,1]$ is as easy as that of $\sigma(x)$ for real $x$. Let $u_{n}(\theta), l_{n}^{\prime}(\theta)$ be the left, right singular vectors of $\left[A-\left(x_{k}-\theta p_{k 1}+y_{k} \mathrm{i}-\theta p_{k 2} \mathrm{i}\right) I, B\right]$ corresponding to $\sigma\left(x_{k}-\theta p_{k 1}, y_{k}-\theta p_{k 2}\right)$, then $f(\theta)=v_{n}^{\mathrm{H}}(\theta)\left({ }_{0}^{u_{1}^{\prime \prime}(\theta)}\right)$ is well defined. We introduce

$$
g(\theta)=\frac{\mathrm{d} \sigma}{\mathrm{d} \theta}\left(x_{k}-\theta p_{k 1}, y_{k}-\theta p_{k 2}\right),
$$

then $g(\theta)$ has the following expression by (2.10),

$$
g(\theta)=p_{k 1} \operatorname{Re} f(\theta)+p_{k 2} \operatorname{Im} f(\theta) .
$$


As a direct consequence of Theorem $1, \dot{g}(\theta)$ is given by

$$
\dot{g}(\theta)=p_{k 1} \operatorname{Re} \dot{f}(\theta)+p_{k 2} \operatorname{Im} \dot{f}(\theta) .
$$

All together we have the following algorithm to calculate $\theta_{k}$ :

Newton Algorithm to compute $\theta_{k}$. (a) Initial value $\theta_{0}=1$.

(b) Run the following Newton method for $j=1,2, \ldots$ :

$$
\theta_{j+1}=\theta_{j}-\eta_{j} \frac{g\left(\theta_{j}\right)}{\dot{g}\left(\theta_{j}\right)},
$$

where $\eta_{j}$ is chosen such that $\sigma\left(x_{k}-\theta_{j+1} p_{k 1}, y_{k}-\theta_{j+1} p_{k 2}\right)<\sigma\left(x_{k}-\theta_{j} p_{k 1}, y_{k}-\theta_{j} p_{k 2}\right)$.

Also the bisection method can be used to find the zeros of $g(\theta)$. Numerical results suggest that this Newton's method with the parameter $\theta_{k}$ enjoys the property of global convergence. Moreover one needs only to compute two or three $\theta_{k}$ 's to get a good initial point for the Newton method. It means that after two or three steps $\theta_{k}$ will be near to 1 . Thus Newton's method with $\theta_{k}=1$ will converge quadratically. Hence $\theta_{k}$ is only calculated in the first three steps, it is automatically taken to be 1 since then. Computing the minimum $\theta_{k}$ takes much work. One needs generally seven or eight SVDs to find a good approximate value to $\theta_{k}$. However it seems that this step cannot be neglected. It is worthwhile to say that there exists only one zero point of $g(\theta)$ in our examples.

Another way of selecting $\theta_{k}$ is from the following inequality:

$$
\left\|u_{n}^{\mathrm{H}}(0)\left[A-\left(x_{k}+\mathrm{i} y_{k}\right) I, B\right]\right\| \geq\left\|u_{n}^{\mathrm{H}}(0)\left[A-\left(x_{k}-\theta_{k} p_{k 1}+y_{k} \mathrm{i}-\theta_{k} p_{k 2} \mathrm{i}\right) I, B\right]\right\|,
$$

where $\theta_{k}=-\left\{\sigma\left(x_{k}, y_{k}\right)\left(p_{k 1} \operatorname{Re} f(0)+p_{k 2} \operatorname{Im} f(0)\right)\right\} /\left(p_{k 1}^{2}+p_{k 2}^{2}\right)$. From this inequality, we have

$$
\sigma\left(x_{k}, y_{k}\right) \geq \sigma\left(x_{k}-\theta_{k} p_{k 1}, y_{k}-\theta_{k} p_{k 2}\right) \text {. }
$$

But Newton's method with $\theta_{k}$ selected in this way converges to $s^{*}$ very slowly.

Before we finish this section, we shall discuss how to select a good initial point for the Newton method. In [3], it has been proven that $s^{*}$ is located within one of the disks in the complex plane whose centers are the eigenvalues of $F$, where

$$
F=\left(\begin{array}{ll}
A & B \\
C & D
\end{array}\right),
$$

with $[C, D]$ a random matrix or having orthogonal rows such that $F$ is square. Our numerical examples show that those disks are small and almost located in the region (4.1). So the eigenvalues of $F$ and $A$ are generally a good choice of the initial points. For our examples, Newton's algorithm with the initial points being the eigenvalues of $F$ without selecting the parameters $\theta_{k}$ (i.e. $\theta_{k}=1$ ), converges to the local minima of $\sigma_{n}(s)$ within 5 steps.

\section{Numerical examples}

Two examples presented in [12] are implemented under MATLAB. Using Newton's method and the bisection method, all minimum points of $\sigma(s)$ with $s$ being real are found. So there exists no difficulty to get $d_{\mathrm{r}}(A, B)$. But for $d(A, B)$, though we have known the exact region containing all zero points of $f(s)$, the number of zero points is still a problem. Generally speaking, the Newton's method converges very quickly if a good initial point is selected. 
Example 1. Consider

$$
A=\left(\begin{array}{rrrrr}
3.28 & -2.44 & -1.54 & -3.20 & -3.34 \\
-1.58 & -1.02 & 3.86 & 4.15 & 3.94 \\
-4.06 & 3.54 & 1.65 & 1.79 & 2.15 \\
-4.15 & 3.96 & 0.84 & -2.70 & -2.70 \\
-1.76 & 0.29 & -1.14 & -1.64 & -2.21
\end{array}\right), \quad B=\left(\begin{array}{r}
-2.80 \\
2.79 \\
1.88 \\
-0.48 \\
-1.89
\end{array}\right)
$$

For this example, we compute $d_{\mathrm{r}}(A, B)$, so $f(s)$ will be real. All minimal points of $\sigma(s)$ are according to (4.2) in the region

$$
(-8.5123,9.7310) \text {. }
$$

The graphs of $f(s)$ and $\sigma(s)$ are shown in Figure 1. One can see that the minima and maxima of $\sigma(s)$ are interlacing. $f(s)$ has seven zero points including four minima. When $s^{*}=0.431388, \sigma=0.231910$ is the minimum value. So $s^{*}$ will minimize $d_{\mathrm{r}}(A, B)=\min _{s \in R} \sigma(s)$ and $d_{\mathrm{r}}(A, B)=0.231910$. In [12], only two zero points of $f(s)$ are found. Neither of them reaches the value of $d_{\mathrm{r}}(A, B)$. When taking any point in the interval $(-8.5123,9.7310)$ as an initial point, the Newton algorithm (real case) converges to a minimum point of $\sigma(s)$ within 5 steps. $\left|s_{k}-s^{*}\right| \leq 10^{-6}$, where $s_{k}$ is the acceptable iterative value of the Newton's method. When the initial values are taken as the eigenvalues of $F$ defined in (4.4), the Newton method converges to the local minimum points within 5 steps without selecting $\theta_{k}\left(\theta_{k}=1\right)$.

Example 2. Consider

$$
A=\left(\begin{array}{lrr}
1 & 1 & 1 \\
0.1 & 3 & 5 \\
0 & -1 & -1
\end{array}\right), \quad B=\left(\begin{array}{l}
1 \\
0.1 \\
0
\end{array}\right) .
$$

All zero points $s^{*}=x^{*}+\mathrm{i} y^{*}$ are in the rectangular region given by

$$
-1.851295 \leq x \leq 3.992519, \quad-3.074491 \leq y \leq 3.074491 \text {. }
$$

$f(s)$ has only one real zero point $s_{\mathrm{r}}^{*}=1.027337$ and $d_{\mathrm{r}}(A, B)=0.172460$. The minimum point $s^{*}=$ $0.937084+0.998571 \mathrm{i}$ minimizes $\min _{s \in C} \sigma(s)$ and $d(A, B)=0.039238$. We tried several initial points, the
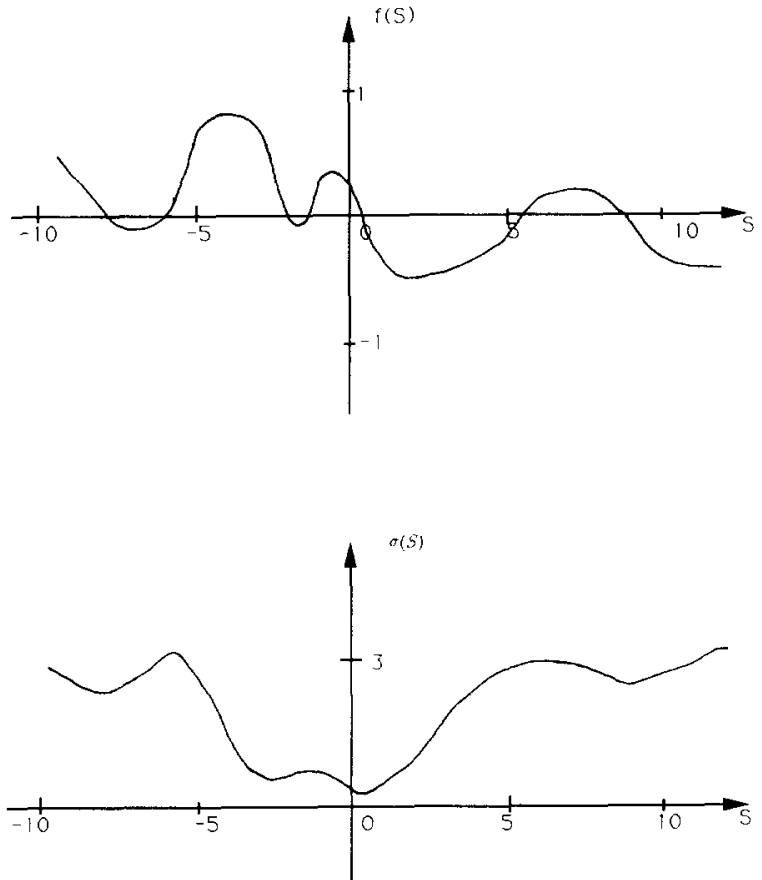

Fig. 1. 


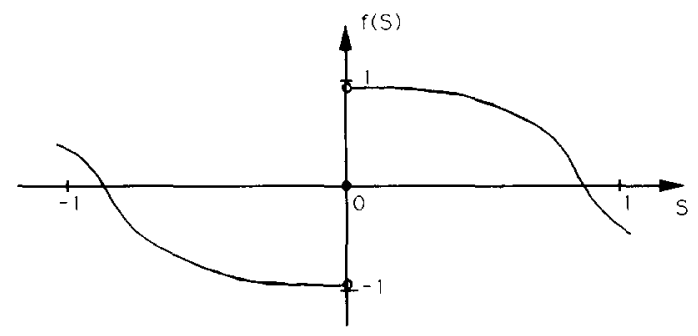

Fig. 2.

Newton method with each of them converges to $s^{*}$ in 5 steps. Say $s_{0}=1.5+\mathrm{i}$, we found $\theta_{0}=0.09935$, $\theta_{1}=0.564158$ and $\theta_{2}=1.00126$. So from step $3 \mathrm{on}, \theta_{k}=1$ is selected. However if the initial point is taken as one of the eigenvalues of $F$ in (4.4), say $0.8625+0.9749 \mathrm{i}$, then the Newton's algorithm with $\theta_{k}=1$ converges to $s^{*}$ in 5 steps.

Example 3. Consider

$$
A=\left(\begin{array}{cccc}
0 & 1 & & \\
& \ddots & \ddots & \\
& & 0 & 1 \\
& & & 0
\end{array}\right), \quad B=\left(\begin{array}{c}
0 \\
\vdots \\
0 \\
1
\end{array}\right) .
$$

In this example, $A$ is a $10 \times 10$ matrix, $B$ a $10 \times 1$ matrix. Let $f(s)$ be the real function of the real parameter $s$, then $f(s)$ has three zero points in real axis. They are 0 and $-0.959492,+0.959492$. It is interesting to know that $f(s)$ is no longer a continuous function. It has a big jump at zero point (see Figure 2).

The reason is that $[A-s I, B]$ has a multiple least singular value 1 at $s=0$, so its singular vector $u_{n}(s)$ may not be continuous at zero. In such case, one can change the initial point to run the Newton algorithm again. Fortunately this extreme case never happens at $s^{*}$.

\section{Multiple singular values and conclusions}

Our theorems about the differentiability of the least singular value $\sigma_{n}(s)$ of $G(s)$, a matrix with $\operatorname{Re}(G(s))$ and $\operatorname{Im}(G(s))$ being real analytic matrix-valued function of a real parameter $s$, are based on the assumption of $\sigma_{n}(s)$ being simple. In this section, we discuss the case of $\sigma_{n}(s)$ being a multiple singular value of $G(s)$. The problem of minimizing the least singular value of $G(s)$ is very different from that of minimizing the largest one of $G(s)$. The solution of the latter is usually at a point where singular values coalesce, i.e. at a nondifferentiable point, since the minimization will drive several singular values to the same minimum value [8]. But for the former problem, its local minimum does not happen at the cross singular values in general. So at its local minimum point, $\sigma_{n}(s)$ is generally simple and differentiable.

When the minimum of $\sigma_{n}(s)$ happens at the point $s^{*}$ (this is extremely unusual), where $\sigma_{n-r+1}\left(s^{*}\right)=$ $\cdots=\sigma_{n}\left(s^{*}\right), \sigma_{n}(s)$ is also differentiable at $s^{*}$, and its derivative is zero. This comes from the fact that the left and right limits of $\dot{\sigma}_{n}(s)$ at $s^{*}$ always exist [11], and they are equal to zero when $s^{*}$ is a local minimum point (see the graph below). What about the second derivative of $\sigma_{n}(s)$ at $s *$ ? We claim that the second derivative of $\sigma_{n}(s)$ at $s^{*}$ always exists too. Considering $\sigma_{j}(s)$ and $\sigma_{n}(s)$, where $j$ is one number of $\{n-r+1, \ldots, n-1\}$, we define two new functions $p_{1}(s)$ and $p_{2}(s)$ near $s^{*}$ as the original singular value functions without ordering them, so $p_{1}(s)$ and $p_{2}(s)$ are analytic near $s^{*}$. The relations of $p_{1}(s), p_{2}(s)$ and $\sigma_{j}(s)$ and $\sigma_{n}(s)$ are $\sigma_{j}(s)=\max \left\{p_{1}(s), p_{2}(s)\right\}$ and $\sigma_{n}(s)=\min \left\{p_{1}(s), p_{2}(s)\right\}$. Moreover $p_{1}\left(s^{*}\right)=p_{2}\left(s^{*}\right)$ and $\dot{p}_{1}\left(s^{*}\right)=\dot{p}_{2}\left(s^{*}\right)=0$, since $\sigma_{j}\left(s^{*}\right)=\sigma_{n}\left(s^{*}\right)$ and $\dot{\sigma}_{j}\left(s^{*}\right)=\dot{\sigma}_{n}\left(s^{*}\right)=0$. Let us assume 


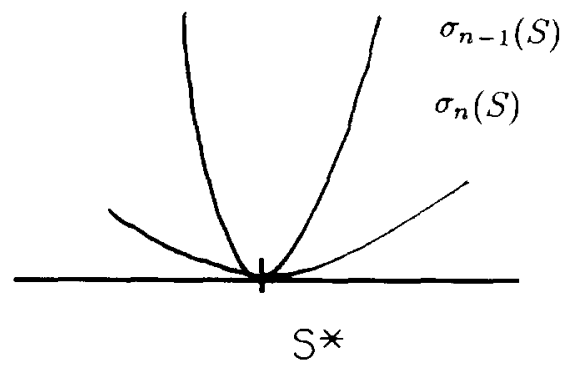

Fig. 3.

that $\left(\mathrm{d}^{2} p_{1} / \mathrm{d} s^{2}\right)\left(s^{*}\right) \geq\left(\mathrm{d}^{2} p_{2} / \mathrm{d} s^{2}\right)\left(s^{*}\right)$. When $\left(\mathrm{d}^{2} p_{1} / \mathrm{d} s^{2}\right)\left(s^{*}\right)>\left(\mathrm{d}^{2} p_{2} / \mathrm{d} s^{2}\right)\left(s^{*}\right)$, we have $p_{1}(s)-p_{2}(s)$ $\geq 0$ near to $s^{*}$. By the definitions of $p_{1}(s)$ and $p_{2}(s)$, we know that $p_{1}(s)=\sigma_{i}(s)$ and $p_{2}(s)=\sigma_{n}(s)$, so $\sigma_{n}(s)$ is analytic. When $\left(\mathrm{d}^{2} p_{1} / \mathrm{d} s^{2}\right)\left(s^{*}\right)=\left(\mathrm{d}^{2} p_{2} / \mathrm{d} s^{2}\right)\left(s^{*}\right)$, we know that the second derivative of $\sigma_{n}(s)$ exists near $s^{*}$. Hence the Newton method can be used at the minimum points of $\sigma_{n}\left(s^{*}\right)$. See Figure 3 .

We have presented a new method to compute the distance to uncontrollability $d(A, B)$, which is based on the explicit expressions of the first and second derivatives of $\sigma(x, y)$. Numerical examples show that this method works well.

\section{References}

[1] D.L. Boley and W. Lu, Measuring how far a controllable system is from an uncontrollable one, IEEE Trans. Automat. Control 31 (1986) 249-251.

[2] D.L. Boley, Computing rank-deficiency of rectangular matrix pencials, Systems Control Lett. 9 (1987) $207-214$.

[3] D.L. Boley, Estimating the sensitivity of the algebraic structure of pencils with simple eigenvalue estimates, SLAM J. Matrix Anal. Appl. (Oct. 1990).

[4] R. Byers, Detecting nearly uncontrollable pairs, Proceedings of the International Symposium MTNS-89 (Amsterdam, 1989).

[5] R. Eising. The distance between a system and the set of uncontrollable systems, Proc. MTNS, Beer-sheva, June 1983 (Springer-Verlag, Berlin-New York, 1984) 303-314.

[6] R. Eising, Between controllable and uncontrollable, Systems Control Lett. 4 (1984) 263-264.

[7] C. Kenney and A.J. Laub, Controllability and stability radii for companion form systems, Math. Control Signals Systems (1988).

[8] M.L. Overton, On minimizing the maximum eigenvalue of a symmetric matrix, SIAM J. Matrix Anal. Appl. 9 (1988) 256-268.

[9] C.C. Paige, Properties of numerical algorithms relating to controllability, IEEE Trans. Automat. Control 26 (1981) $130-138$.

[10] Ji-guang Sun, A note on simple non-zero singular values, J. Comput. Math. 6 (1988) 259-266.

[11] Ji-guang Sun, Sensitivity analysis of zero singular values and multiple singular values J. Comput. Math. 6 (1988) $325-335$.

[12] M. Wicks and R. DeCarlo, Computing the distance to uncontrollable system, IEEE Trans. Automat. Control (to appear).

[13] J.H. Wilkinson, The Algebraic Eigentalue Problem (Clarendon Press, Oxford, 1965). 